Draft version November 20, 2018

Preprint typeset using $\mathrm{L}_{\mathrm{T}} \mathrm{EX}$ style emulateapj v. 11/10/09

\title{
THE FRONTIER BETWEEN SMALL-SCALE BIPOLES AND EPHEMERAL REGIONS IN THE SOLAR PHOTOSPHERE: EMERGENCE AND DECAY OF AN INTERMEDIATE-SCALE BIPOLE OBSERVED WITH IMAX/SUNRISE
}

\author{
S. L. Guglielmino ${ }^{1,2}$, V. Martínez Pillet ${ }^{1}$, J. A. Bonet ${ }^{1,2}$, J. Carlos del Toro Iniesta ${ }^{3}$, L. R. Bellot Rubio ${ }^{3}$, \\ S. K. Solanki ${ }^{4,5}$, W. Schmidt ${ }^{6}$, A. Gandorfer ${ }^{4}$, P. Barthol $^{4}$, M. Knölker $^{7}$ \\ Draft version November 20, 2018
}

\begin{abstract}
We report on the photospheric evolution of an intermediate-scale $(\approx 4 \mathrm{Mm}$ footpoint separation $)$ magnetic bipole, from emergence to decay, observed in the quiet Sun at high spatial $\left(0^{\prime \prime} \cdot 3\right)$ and temporal $(33 \mathrm{~s})$ resolution. The observations were acquired by the IMaX imaging magnetograph during the first science flight of the SUNRISE balloon-borne solar observatory. The bipole flux content is $6 \times 10^{17} \mathrm{Mx}$, representing a structure bridging the gap between granular scale bipoles and the smaller ephemeral regions. Footpoints separate at a speed of $3.5 \mathrm{~km} \mathrm{~s}^{-1}$ and reach a maximum distance of $4.5 \mathrm{Mm}$ before the field dissolves. The evolution of the bipole is revealed to be very dynamic: we found a proper motion of the bipole axis and detected a change of the azimuth angle of $90^{\circ}$ in 300 seconds. The overall morphology and behaviour are in agreement with previous analyses of bipolar structures emerging at granular scale, but we also found several similarities with emerging flux structures at larger scale. The flux growth rate is $2.6 \times 10^{15} \mathrm{Mx} \mathrm{s}^{-1}$, while the mean decay rate is one order of magnitude smaller. We describe in some detail the decay phase of the bipole footpoints which includes break up into smaller structures, interaction with pre-existing fields leading to cancellation but appears to be dominated by an as-yet unidentified diffusive process that removes most of the flux with an exponential flux decay curve. The diffusion constant $\left(8 \times 10^{2} \mathrm{~km}^{2} \mathrm{~s}^{-1}\right)$ associated with this decay is similar to the values used to describe the large scale diffusion in flux transport models.
\end{abstract}

Subject headings: Sun: magnetic topology — Sun: photosphere — Sun: activity

\section{INTRODUCTION}

Magnetic flux emergence in the solar photosphere involves a variety of spatial scales. It spans from the small bipoles with fluxes of the order of $10^{16} \mathrm{Mx}$ (Martínez González \& Bellot Rubio 2009; Danilovic et al. 2010), that populate the quiet Sun at any stage of the solar activity cycle, to the large, complex active regions present during solar maximum, with fluxes of the order of $10^{22} \mathrm{Mx}$ (Lites et al. 1998).

Bipolar structures with absolute flux content ranging from $10^{18} \mathrm{Mx}$ to $5 \times 10^{19} \mathrm{Mx}$ are usually referred to as ephemeral regions (ERs), due to the short lifetime of less than 24 hours that was estimated when they were first discovered (Harvey \& Martin 1973). The opposite polarity components of ERs separate from each other during the growth period, reaching a distance of a few megameters in about half an hour with decreasing separation velocity (Martin 1988). Then, ERs decay in a complex way, strongly dependent upon the surrounding magnetic

sgu@iac.es

${ }^{1}$ IAC Instituto de Astrofísica de Canarias, C/ Vía Láctea s/n, La Laguna, Tenerife, E-38200, Spain

2 ULL Departamento de Astrofísica, Univ. de La Laguna, La Laguna, Tenerife, E-38205, Spain

3 IAA Instituto de Astrofísica de Andalucía (CSIC), Apdo. de Correos 3004, E 18080 Granada, Spain

4 MPS Max-Planck-Institut für Sonnensystemforschung, MaxPlanck-Str. 2, 37191 Katlenburg-Lindau, Germany

5 School of Space Research, Kyung Hee University, Yongin, Gyeonggi 446-701, Korea

6 KIS Kiepenheuer-Institut für Sonnenphysik, Schöneckstr. 6, 79104 Freiburg, Germany

7 HAO High Altitude Observatory, National Center for Atmospheric Research, P.O. Box 3000, Boulder, CO 80307-3000, USA network elements.

This evolution scenario was confirmed by the study of Hagenaar (2001), who analyzed a large statistical sample of ERs using time series of magnetograms taken with the Michelson Doppler Imager (MDI) aboard the $S O H O$ satellite. She found an average lifetime of $\simeq 4$ hours, an average separation of $\simeq 9 \mathrm{Mm}$, depending on the total amount of magnetic flux carried by the ER, and a separation velocity decreasing from $4 \mathrm{~km} \mathrm{~s}^{-1}$ to $1.5 \mathrm{~km} \mathrm{~s}^{-1}$. The flux concentrations fragmented and moved apart, merging with other concentrations or with intranetwork fields.

The origin of ERs is quite controversial. They could represent the small-scale tail of the distribution of active regions, generated by the large scale solar dynamo (Parker 1955), as argued by Harvey et al. (1975), or be generated by a near-surface local dynamo (Nordlund et al. 1992; Parker 1993; Cattaneo 1999). Alternatively, they might be the end product of failed active region emergence processes that end in a catastrophic explosion (Moreno Insertis et al. 1995).

To shed light into their origin, Hagenaar (2001) also analyzed the relationship of ERs with the solar magnetic activity, concluding that only $60 \%$ of the ERs had an orientation consistent with Hale's polarity law. Hagenaar et al. (2003) found that the number of ERs varies by a factor of 1.5 in antiphase with the solar cycle, confirming the early findings of Martin \& Harvey (1979), and that the latitude distribution of ERs is broader than that of the activity belts. The frequency spectrum of ERs deduced by Hagenaar et al. (2003) appeared to be a continuous, smoothly decreasing distribution of bipolar 
flux regions, formed by two distinct but coupled powerlaw spectra, with a turnover at around $2 \times 10^{19} \mathrm{Mx}$. This suggested the coexistence of both dynamo mechanisms, the global dynamo producing cycle-modulated large ERs and active regions, and the local dynamo generating small-scale bipoles independent of the cycle phase. Furthermore, Hagenaar et al. (2008) showed that the rate of ERs emergence depends on the local flux imbalance: it is lower within strongly unipolar regions by a factor of 3 relative to flux-balanced areas in the quiet Sun. This indicated that a large background field may affect the ERs generation process in the sub-surface layers.

In the meantime, our knowledge of small-scale magnetic fields greatly improved from below the ERs threshold at $10^{18} \mathrm{Mx}$ down to $10^{16} \mathrm{Mx}$, at the limit of current detection capabilities. Thanks to the highresolution observations taken by both ground-based telescopes with adaptive optics, and the Solar Optical Telescope (SOT; Tsuneta et al. 2008) aboard the Hinode satellite (Kosugi et al. 2007), a number of small low-flux bipolar structures have been studied in recent years. Several contributions reported on the emergence of horizontally inclined magnetic fields with strengths of a few hundred $G$ in the quiet Sun, mostly at granular scale (Martínez González et al. 2007; Centeno et al. 2007; Orozco Suárez et al. 2008; Lites et al. 2008; Ishikawa et al. 2008; Martínez González \& Bellot Rubio 2009; Gömörv et al. 2010; Ishikawa et al. 2010; Danilovic et al. 2011). The usual evolution of these structures begins with the appearance of an isolated patch of linear polarization signals, indicating horizontal fields, followed by the appearance of opposite-polarity circular polarization signals at opposite edges of the horizontal patch. These results are often interpreted as the rise of small-scale magnetic loops through the photosphere.

Different mechanisms have been proposed to explain the decay of the bipoles: submergence of $\Omega$-loops driven by convective downflows, flux dispersal resulting from horizontal convective motions with the Stokes signals falling below the instrumental sensitivity, ohmic dissipation, and the rise of U-loops towards the upper photospheric layers following reconnection below the solar surface.

Parnell et al. (2009) studied MDI and SOT data to determine the flux distribution of all magnetic features that can be observed with these instruments on the solar surface at any one time. They found that flux concentrations can be described by a single power-law distribution with a slope of -1.85 for fluxes between $2 \times 10^{17} \mathrm{Mx}$ to $10^{23}$ $\mathrm{Mx}$. These authors argue that the differences in flux distributions found in previous works result from counting and identifying the magnetic structures in different ways, i.e., from selection effects. Their findings support a scale invariance of the distribution of fluxes that conceivably may extend to even smaller magnetic features beyond the current detection limit. Such a distribution might be produced either (i) by a solar dynamo that operates in the same way at a continuum of scales, and whose length scale increases with depth or (ii) if the magnetic features are generated by distinct dynamos with different characteristic lengths, by reprocessing of flux at the surface. In the latter case, such a process, through merging, cancellation, and fragmentation, would dominate to produce a power-law distribution.

Thornton \& Parnell (2011) investigated the distribution of small-scale intranetwork concentrations counting only the features related to emergence events with fluxes smaller than $10^{17} \mathrm{Mx}$. By combining their results with the results on the distributions of the emergence at larger scale, they found a single power law for all emerging features with fluxes ranging from $10^{16} \mathrm{Mx}$ to $10^{23} \mathrm{Mx}$ with a slope of -2.7 , which is consistent with the findings of Hagenaar et al. (2003). The emergence rate is dominated by small-scale fields, in agreement with Socas-Navarro \& Sánchez Almeida (2002). Since the single power-law distribution is already found at emergence, before the surface flux reprocessing, this result is suggestive of the idea that dynamo action occurs on all scales. Large magnetic structures would be created in the tachocline, whilst the smaller features would continuously occur by a turbulent dynamo action over a range of scales throughout the convection zone. $\mathrm{Nu}-$ merical convection simulations also support this scenario (Stein et al. 2010). The steeper slope of emerging structures $(-2.7)$ with respect to the magnetic features present at any instant on the solar surface $(-1.85)$ can be explained taking into account the shorter lifetime of the small-scale fields and their coalescence at the edges of the supergranules.

In this paper, we report on the emergence of a magnetic bipole, observed at high spatial resolution by the Imaging Magnetograph eXperiment (IMaX; Martínez Pillet et al. 2011) mounted on the 1-m aperture telescope flown on board the SUNRISE balloon-borne solar observatory (Solanki et al. 2010; Barthol et al. 2011; Berkefeld et al. 2011; Gandorfer et al. 2011). We present a high-cadence analysis of the temporal evolution of this magnetic structure. The flux content of the bipole is $\sim 6 \times 10^{17} \mathrm{Mx}$, which places it close to the limit between the ERs and the small-scale fields observed at granular scale. In Sect. 2 we describe the observations, in Sect. 3 we report our results on both the emergence and the decay phases, and in Sect. 4 we discuss our findings in a general context.

\section{OBSERVATIONS AND DATA ANALYSIS}

We have analyzed an IMaX data set obtained on 2009 June 9, 01:30:54-02:02:29 UT. During that time, IMaX took polarization maps at five wavelength positions over the Fe I $525.02 \mathrm{~nm}$ line (Landé factor $g=3$ ) at $\lambda=-8$, $-4,+4,+8$, and $+22.7 \mathrm{pm}$ from the line center, with a spectral resolution of $8.5 \mathrm{pm}$. Six images were accumulated per wavelength point, and the full Stokes vector was recorded (V5-6 mode). The temporal cadence of the scans is $33 \mathrm{~s}$, with a pixel size of 0 '. 055 . Figure 1 shows the field of view (FoV) covered by the observations, about $50^{\prime \prime} \times 50^{\prime \prime}$ over a quiet region at the disk center.

All data have been corrected for instrumental effects, by performing dark-current subtraction, flat-field correction, and cross-talk removal. The blueshift over the FoV due to the collimated setup of the Fabry-Pérot etalon of the magnetograph is corrected in the inferred velocity values. Two different types of data are produced: non-reconstructed data (level 1) and reconstructed data (level 2), obtained by using phase-diversity information. The reconstruction requires an apodization that effectively reduces the IMaX FoV down to about $45^{\prime \prime} \times 45^{\prime \prime}$. In this study, we analyze level 1 data, since the emerging 


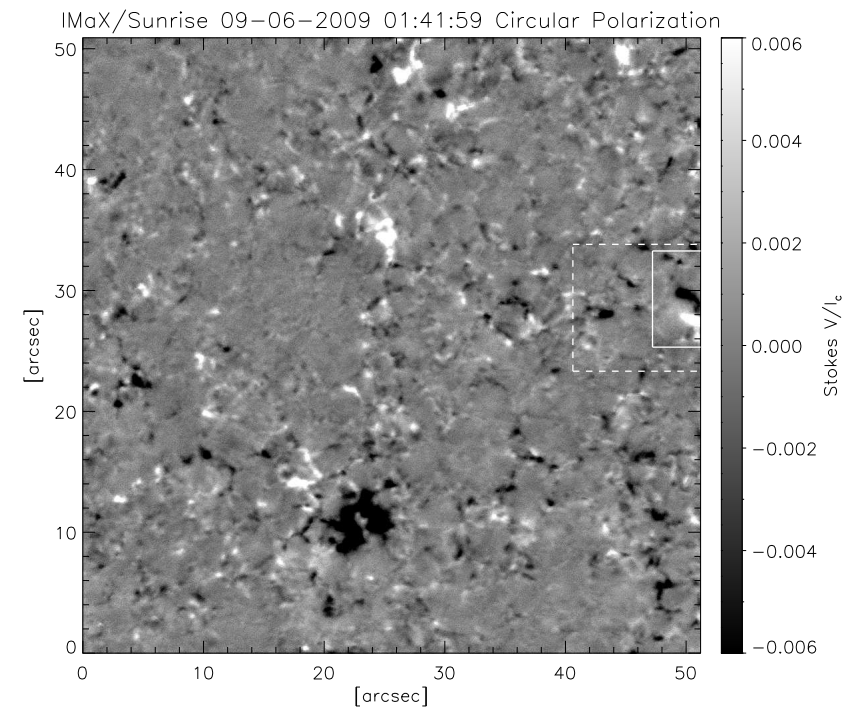

Figure 1. Map of the non-reconstructed mean circular polarization signal $V_{s}$, i.e., the LOS magnetic flux, covering the full FoV of IMaX of about $50^{\prime \prime} \times 50^{\prime \prime}$, obtained during the observations of the emerging bipole analyzed in this work. The square (dashed line), with a FoV of $10^{\prime \prime} 5 \times 10^{\prime \prime} 5$, indicates the sub-region whose spectra have been inverted with the SIR code. The inner rectangle (solid line), with a FoV of $\sim 4^{\prime \prime} \times 8^{\prime \prime}$ indicates the location of the emerging flux region, as shown in the sequences displayed in Fig. 3

bipole was observed just at the right border of the full FoV (within the box in Fig. 11). The spatial resolution is 0 "'3 (before reconstruction) and the noise level is about $1 \times 10^{-3}$ in units of continuum intensity per wavelength point in each Stokes parameter. Further details about data reduction are provided by Martínez Pillet et al. (2011).

We obtained maps of the mean circular polarization averaged over the line, $V_{s}$, and of the mean linear polarization signal $L_{s}$, given respectively by

$$
V_{s}=\frac{1}{4\left\langle I_{c}\right\rangle} \sum_{i=1}^{4} \epsilon_{i}\left|V_{i}\right|
$$

and

$$
L_{s}=\frac{1}{4\left\langle I_{c}\right\rangle} \sum_{i=1}^{4} \sqrt{{Q_{i}}^{2}+U_{i}{ }^{2}},
$$

where $\left\langle I_{c}\right\rangle$ is the continuum intensity averaged over the IMaX FoV, $\epsilon=[1,1,-1,-1]$, and $i$ runs over the first four wavelength positions.

We carried out inversions of the observed $\mathrm{Fe}$ I $525.02 \mathrm{~nm}$ Stokes vector spectra using the SIR code (Ruiz Cobo \& del Toro Iniesta 1992). This code numerically solves the radiative transfer equation along the line of sight (LOS) for Zeeman-polarized radiation under the assumption of local thermodynamic equilibrium.

The inversion yields the temperature stratification in the range $-4.0<\log \tau<0$, where $\tau$ is the optical depth of the continuum at $500 \mathrm{~nm}$. We use the Harvard Smithsonian Reference Atmosphere (HSRA; Gingerich et al. (1971) as initial model. The temperature is modified with two nodes. SIR also provides the LOS velocity $v_{L O S}$, the micro-turbulent velocity $v_{\text {micro }}$, the magnetic field strength $B$, the inclination and azimuth angles $\gamma$ and $\phi$ in the LOS reference frame. As these IMaX observations were taken at disk center, the returned magnetic parameters do not need to be converted to local solar coordinates. The magnetic filling factor has been assumed unity for these inversions, not taking into account stray light contamination. The synthetic profiles are convolved with the spectral Point Spread Function at the focal plane of IMaX.

In a first series of inversions, all the physical parameters are assumed to be constant with height, except for the temperature that has two nodes, as already mentioned above. In another series of inversions, $v_{L O S}$ and $B$ are also allowed to have a linear dependence with the optical depth $\tau$. Such a gradient in both parameters has been found to be necessary in order to fit some profiles that show a considerable asymmetry between the blue and red lobes of Stokes $V$, or between the lobes of $Q$ and $U$ when they are significant. Elsewhere the fits obtained without gradient in velocity and magnetic field strength is found to be as good as the one with gradients. The total number of free parameters is 7 for SIR inversions (9 if gradients in $v_{L O S}$ and $B$ are included) and the number of the data points in the V5-6 mode is 20 .

The absolute velocity is calibrated using the results coming from the Gaussian fits obtained for the same data set (see, e.g., Roth et al. 2010), compared with the results of the SIR inversion without gradients. This calibration uses a granular mean blueshift of $200 \mathrm{~m} \mathrm{~s}^{-1}$.

In Fig. 2 we display a subset of the typical profiles acquired by IMaX, and the corresponding fits obtained using the SIR code. The spectra plotted in blue, showing predominantly horizontal fields, and red, with more vertical fields, show profiles well fitted without any gradient. There are also clear examples of spectra showing noticeable asymmetries in Stokes $V$ in absence of a significant $L_{s}$ signal (plotted green), as well as in Stokes $Q$ and $V$ with clear horizontal fields (plotted orange), which both require a gradient in $v_{L O S}$ and $B$. These profiles show either downflows or upflows, respectively, as can be deduced also from the shifts with respect to the line center in Stokes $I$. The residuals of the fit, defined as $P_{o b s}-P_{f i t}$, with $P$ being any of the polarization profiles, show an rms variation over the inverted FoV of $1-1.6 \times 10^{-3}$, in agreement with the estimated noise per wavelength sample of the data.

\section{RESULTS}

\subsection{Flux emergence phase}

Figure 3 shows a temporal sequence of the continuum intensity $I_{c}$, and of the polarization maps $L_{s}$ and $V_{s}$. This sequence tracks the emergence of the bipole since its first detection in the photosphere. Green contours enclose the region with a significant linear polarization signal, i.e. $L_{s}>0.5 \%$, whereas red and blue contours mark areas with positive and negative circular polarization, respectively, with $V_{s}>0.8 \%$. We also provide a movie of the evolution of the bipole in the online journal. The temporal cadence of each frame is $33 \mathrm{~s}$.

At 01:35:54 UT the bipole appears in frame 2 at coordinates $\left[3^{\prime \prime}, 33^{\prime \prime} 5\right]$, inside a pre-existing granule visible at the same location in frame 1 . It is later recognizable as a patch with increasing linear polarization signals in the 

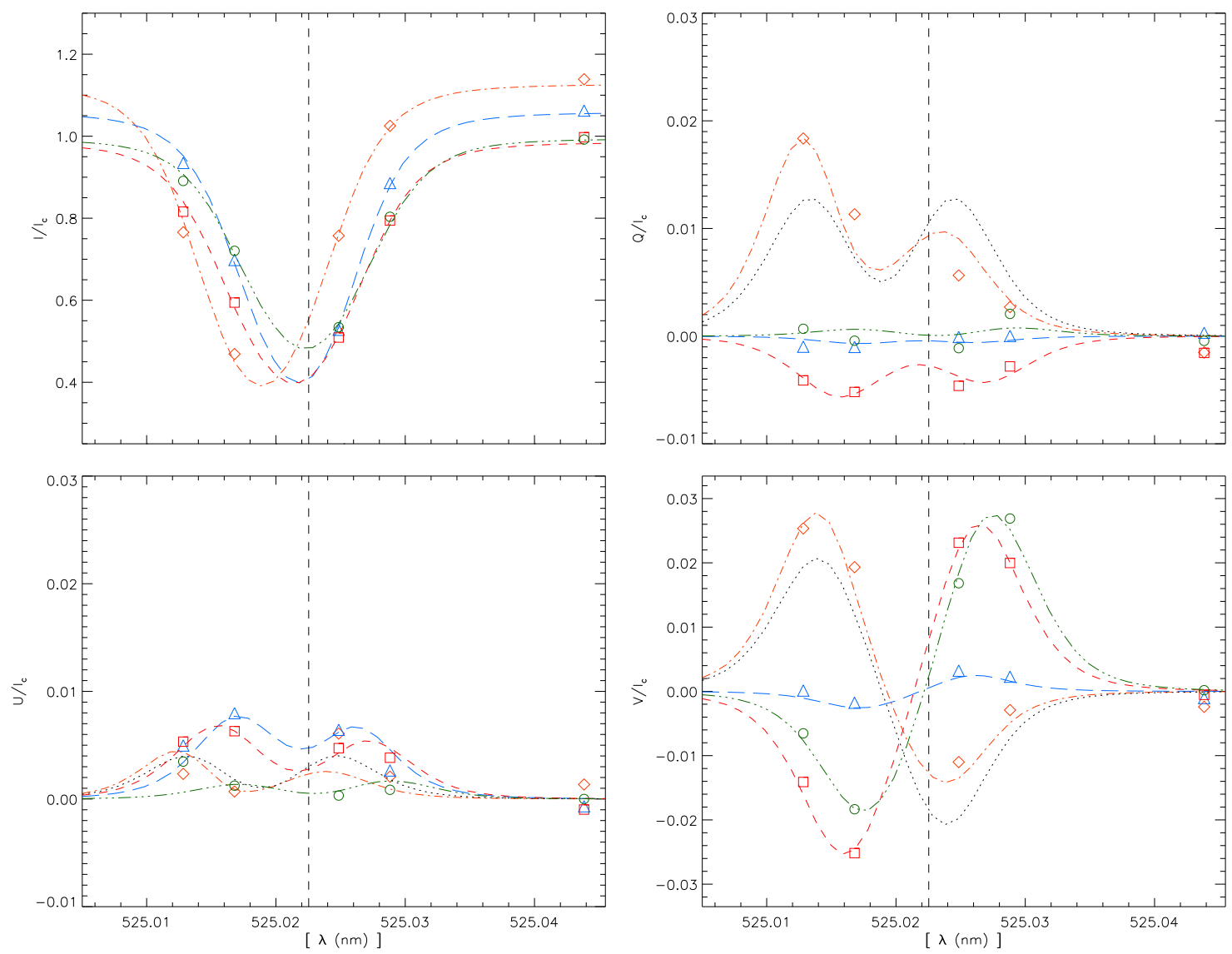

Figure 2. Observed (symbols) $I, Q, U$, and $V$ profiles, for a sample of IMaX spectra, with the corresponding fits (lines) obtained with the SIR code. Blue (triangles/long dashed): horizontal fields, no gradients. Red (squares/dashed): vertical fields, no gradients. Green (circle/dot-dot-dot dashed): asymmetry in Stokes $V$ with weak $L_{s}$ signal, with gradients. Orange (diamonds/dot dashed): asymmetry in the blue and red lobes of Stokes $U$ and $V$, with gradients. The dotted line represents the fit without gradients to the plotted orange spectrum (for Stokes $I$ the dotted line falls on top of the orange one). The dashed vertical line indicates the nominal line center.

following $L_{s}$ maps that always coincides with the top of the granule during the whole emergence process. There is no evidence of any influence of the emerging magnetic flux on the granulation pattern.

Note that the bipole emerges in a region where two subarcsecond bipoles were already present as seen in the first $V_{s}$ frame of Fig. 3 (top panel). One of these bipoles is located at $\left[3^{\prime \prime}, 4^{\prime \prime}\right]$, nearly exactly over the region of emergence of the negative footpoint of our bipole, but with its polarities flipped (i.e., with the positive polarity toward the top part of the figure) with respect to those of the emerging bipole. The other is located at the bottom right of the studied area, at $\left[3 . .5,2^{\prime \prime}\right]$, and possesses the same orientation as our bipole.

The corresponding $V_{s}$ footpoints of the large emerging bipole are first seen in the frames $3-4$, as two small patches of opposite polarities at symmetrically opposite edges of the enhanced $L_{s}$ patches. The evolution of the emerging loop can be easily followed in the subsequent $V_{s}$ frames, where the opposite polarities of the bipole, i.e., the footpoints of the loop, are seen to separate from each other in opposite directions, with quite a strong $L_{s}$ signal in between them being maintained until frame 9 . This implies horizontal field in mid-photospheric layers, which is consistent with continuing flux emergence during this whole period of time.

It is clear that the emerging bipole studied in this work interacts with the two pre-existing ones. The first bipole at $\left[3^{\prime \prime}, 4^{\prime \prime}\right]$ is completely overtaken by the negative polarity footpoint of our larger emerging bipole. The positive polarity footpoint of the new bipole, however, first enters an intergranular region but crosses over it to interact with the negative polarity footpoint of the pre-existing bipole (frame 5 in Fig. [3] at $\left[33^{\prime \prime} 5,2^{\prime \prime} .5\right]$ ). The negative pre-existing footpoint is completely washed away, while the emerging positive footpoint appears to aggregate the positive polarity flux of the pre-existing bipole.

We plot the distance between the footpoints as a function of time in Fig. 4 (left panel), calculated as the distance between the centroids of the areas where $\left|V_{s}\right|$ is greater than $0.5 \%$. The plot displayed in Fig. 4 (left panel) shows that the distance increases linearly during the first $1000 \mathrm{~s}$ of the loop evolution, with a mean separation velocity of $3.5 \mathrm{~km} \mathrm{~s}^{-1}$. Maximum footpoint separation is $4.5 \mathrm{Mm}$. This result has to be compared with the analysis of emerging bipoles carried out by Martínez González \& Bellot Rubio (2009). In some cases, they found an initial velocity separation of the footpoints of $6 \mathrm{~km} \mathrm{~s}^{-1}$, with a change after $500 \mathrm{~s}$ to $\approx 2$ $\mathrm{km} \mathrm{s}^{-1}$. Note that our bipole is comparable to the largest bipoles studied in that work.

The corresponding temporal sequences of the physical parameters retrieved by SIR during the emergence are shown in Fig. [5, for frames $1-8$ of Fig. 3, and in Fig. 6. 

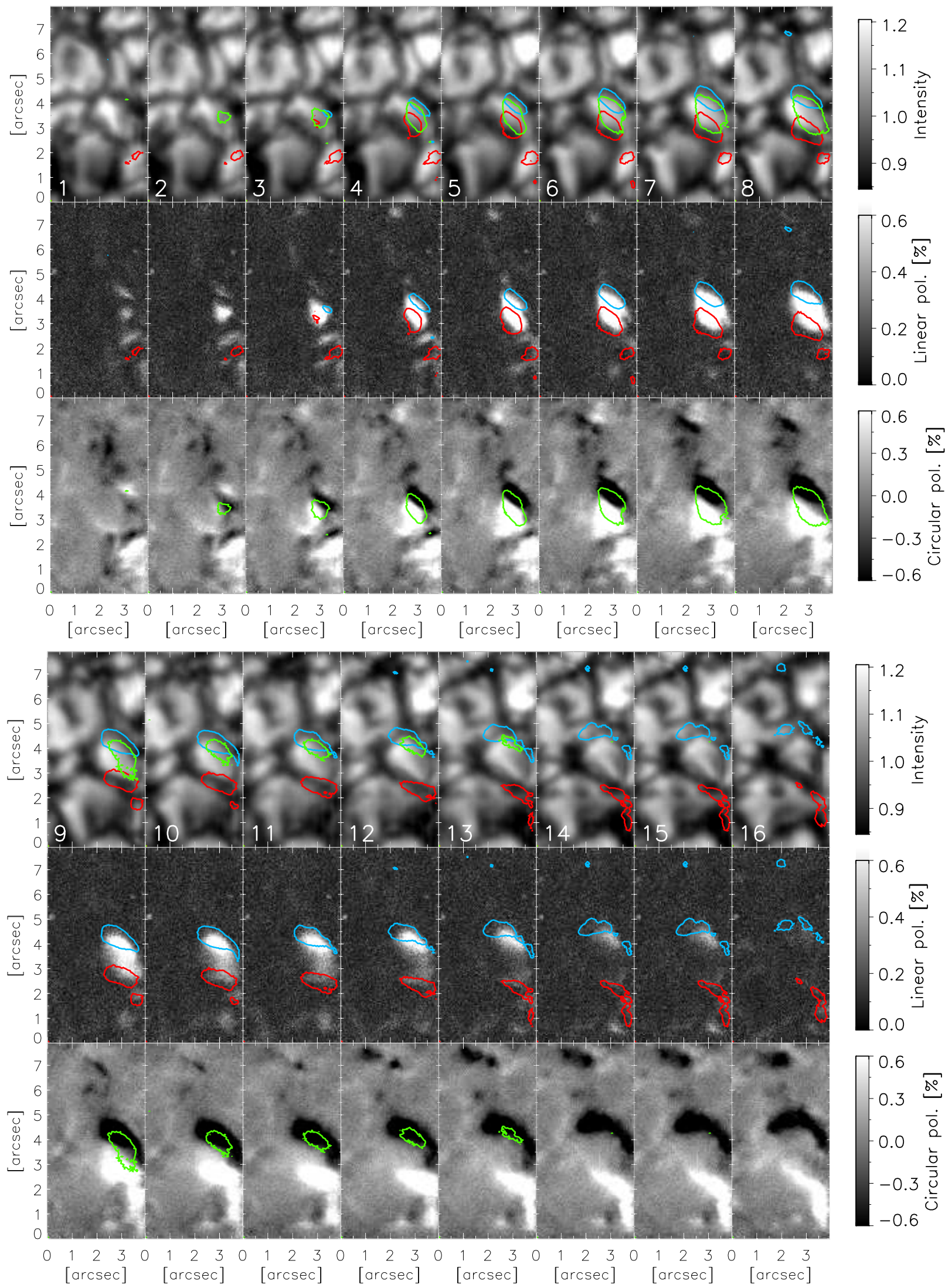

Figure 3. Top rows: Temporal sequence of the continuum maps during the emergence phase of the bipole. Time runs from left to right and continues in the lower set of panels. Red (blue) contours over the maps represent a circular polarization signal of $+0.8(-0.8) \%$ of the $I_{c}$, and green contours represent a linear polarization signal of $0.5 \%$ of the $I_{c}$. Middle rows: the same for the mean linear polarization signal $L_{s}$. Bottom rows: the same for the total circular polarization $V_{s}$. The elapsed time between consecutive frames is $33 \mathrm{~s}$. From frame 11 the decay phase begins. An animation of this figure is available in the online journal. 
for frames $9-16$. We display the values of LOS velocity and of the magnetic field strength averaged between $\log \tau=-1$ and $\log \tau=-2$, where the response functions are more sensitive (note that no filling factor is included in the inversions). Blueshifts, which indicate upward motions, are present in the region since the beginning of the emergence until frame 10 . They are cospatial with the region with strong $L_{s}$ signals, indicative of nearby horizontal fields, and correspond to the granule in the continuum map. The emerging flux region is surrounded by downflows along the intergranular lanes which outline the granule. The larger scale variable velocity pattern near the top of the velocity frames $5-7$ reflects the $p$-mode oscillations, as discussed by, e.g., Roth et al. (2010).

The magnetic field strength has a peak of about 400 $\mathrm{G}$ around frames $5-6$, then it begins to fade (note that this occurs before the footpoints enter the intergranular lanes, which happens in frame 7). As our filling factor is unity, this value should be taken as a flux-equivalent field strength. The maximum values of $B$ are found in the region where the magnetic field is more horizontal. These strengths are of the order of the typical equipartition field strength $B_{e}$ for granules, given by

$$
\frac{B_{e}{ }^{2}}{2 \mu_{0}}=\frac{1}{2} \rho v,
$$

where $\mu_{0}$ is the magnetic permeability of free space, with $v \approx 2 \mathrm{~km} \mathrm{~s}^{-1}$ and $\rho=3 \times 10^{-4} \mathrm{~kg} \mathrm{~m}^{-3}$ (Ishikawa et al. 2008).

The footpoints do not reach a vertical orientation, but they remain rather inclined at an angle of about $45^{\circ}$ with respect to the vertical. Furthermore, they surround the granule having a crescent-shaped aspect, different from the usual circular shape found in previous observations.

In the emergence zone, the azimuth angle is quite homogeneous at every instant during the rise, with the scatter ranging from $\pm 5^{\circ}$ to $\pm 15^{\circ}$ around the average value. However, the mean value, indicated by arrows in Figs. 5 and 6 , changes with time, rotating counterclockwise $10^{\circ}$ per frame until the end of the emergence 8 . The observed rotation rate is thus $0^{\circ} .3 \mathrm{~s}^{-1}$. In the $L_{s}$ and $V_{s}$ maps in Fig. 3, we can see that the bipole axis also rotates in the same counterclockwise direction. This similar rotation for both the field azimuth angle and the bipole footpoints is compatible with the absence of writhe in the emerging flux tube. The observed evolution of the azimuth, which steadily varies by $\Delta \phi \approx 90^{\circ}$ during the emergence, may be attributed to convective motions, but we cannot rule out the presence of some twist in the structure, unfolding during its ascent.

The total magnetic flux content is given by

$$
\Phi=\sum_{i} B_{i} \cos \gamma_{i} S,
$$

where index $i$ runs over all the pixels of the bipole with the same polarity and $S$ is the area of the Sun covered by a pixel. We note here that our assumption of a filling factor equal unity, while probably affects the values found

\footnotetext{
8 Note here that we do not solve the azimuth ambiguity. The arrow is selected simply by choosing the azimuth direction that points from the positive to the negative footpoint of the bipole
}

for the magnetic field strength, has no impact on the estimate of the magnetic flux density and on the total flux content. In both polarities, the flux grows linearly until a maximum of about $6 \times 10^{17} \mathrm{Mx}$ at frame 8 is reached, 4 minutes after the first detection of the bipole as seen in Fig. 4 (right panel). In this figure, the fluxes were estimated by adding all the contributions from manually selected pixels in the footpoint area of interest. Missing points correspond to non-inverted frames. The flux growth rate is thus $2.6 \times 10^{15} \mathrm{Mx} \mathrm{s}^{-1}$.

Note that the slightly smaller positive flux (red symbols) may be due to a different inclination between the footpoints and, as footpoints move apart, to the fact that the corresponding footpoint is located closer to the right boundary of the observed area. At the end of the emergence process (defined as the maximum in the flux curve), the distance between the footpoints of the bipole is about $1.5 \mathrm{Mm}$, with roughly the same size as a typical granule. All these results are consistent with the emergence of an $\Omega$-shaped magnetic bipole at granular scale.

The maximum distance $D$ attained by the footpoints, $4.5 \mathrm{Mm}$, is roughly in agreement with the linear $\log D / \log \Phi$ relation found by Hagenaar (2001) for ERs, which gives $D \simeq 3.5 \times \Phi^{0.18}=5.5 \mathrm{Mm}$.

Active region flux emergence displays upflows associated with transverse fields at equipartition values and downflows at the footpoints with much larger field strengths (see, e.g., Lites et al. 1998; Solanki et al. 2003). In order to compare this behaviour with our bipolar emergence, we have produced a scatter plot of the magnetic field strength and LOS velocity vs. zenith angle $\gamma$, as shown in Fig. 7 for the SIR inversion without gradients in B and $v_{L O S}$ (frame 12). In that frame, the footpoints of the loop, which correspond to the regions with more vertical field, have also stronger field strengths of about $200-250 \mathrm{G}$ with downflows slightly larger than 1 $\mathrm{km} \mathrm{s}^{-1}$, decreasing with time. As already stated above, it is conceivable that these fields are indeed at equipartition values. In the horizontal field regions, an upflow of $1.0 \mathrm{~km} \mathrm{~s}^{-1}$ is seen. This upward motion also decreases with time. Peak upflows for the transverse fields are seen in frames $7-9$ where they reach $2.5 \mathrm{~km} \mathrm{~s}^{-1}$.

No evidence of asymmetry between the footpoints of the bipole is found in the data in both the field strength and the LOS velocity. However, the linear polarization signal, $L_{s}$ is concentrated mainly at the negative footpoint in frames $10-13$.

\subsection{Flux decay phase}

The observations used in this work allow a detailed analysis of the decay phase of the bipole, the less known stage of the evolution of small bipoles and ephemeral regions. The footpoints of the emerged bipole remain visible until the end of the emergence process and well beyond into the decay phase. This phase can be defined to start at the time when the total measured flux begins to decrease, i.e., from frame 9 onwards. Note that the flux curves in Fig. 4 (right panel) never reach a flat portion where the flux of the footpoints would stay constant. Instead, as soon as the maximum is reached, a mechanism somehow begins to erode the newly emerged flux.

Figure 8, which is the continuation of Fig. 3, shows 

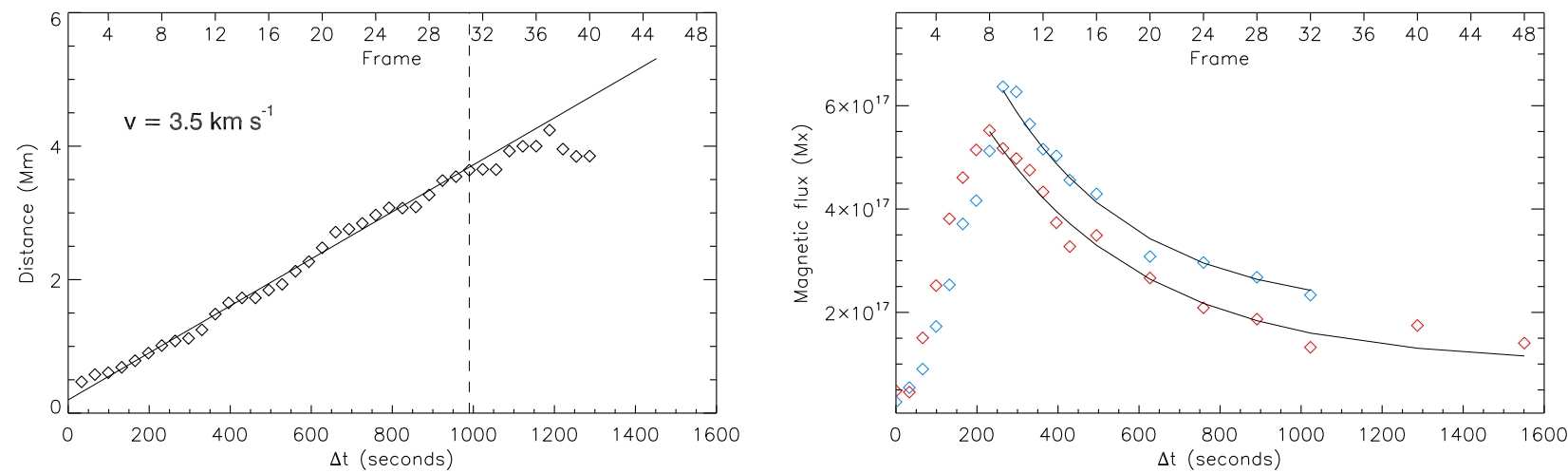

Figure 4. Left panel: Distance between the centroids of the opposite polarity footpoint of the emerging bipole as a function of time. The dotted vertical line indicates the time at which separation deviates from a linear increase. Right panel: Evolution of the flux content in the positive (negative) polarity of the emerging bipole. Red (blue) symbols indicate data values, solid lines represent offset exponential fits to data during the decay phase.

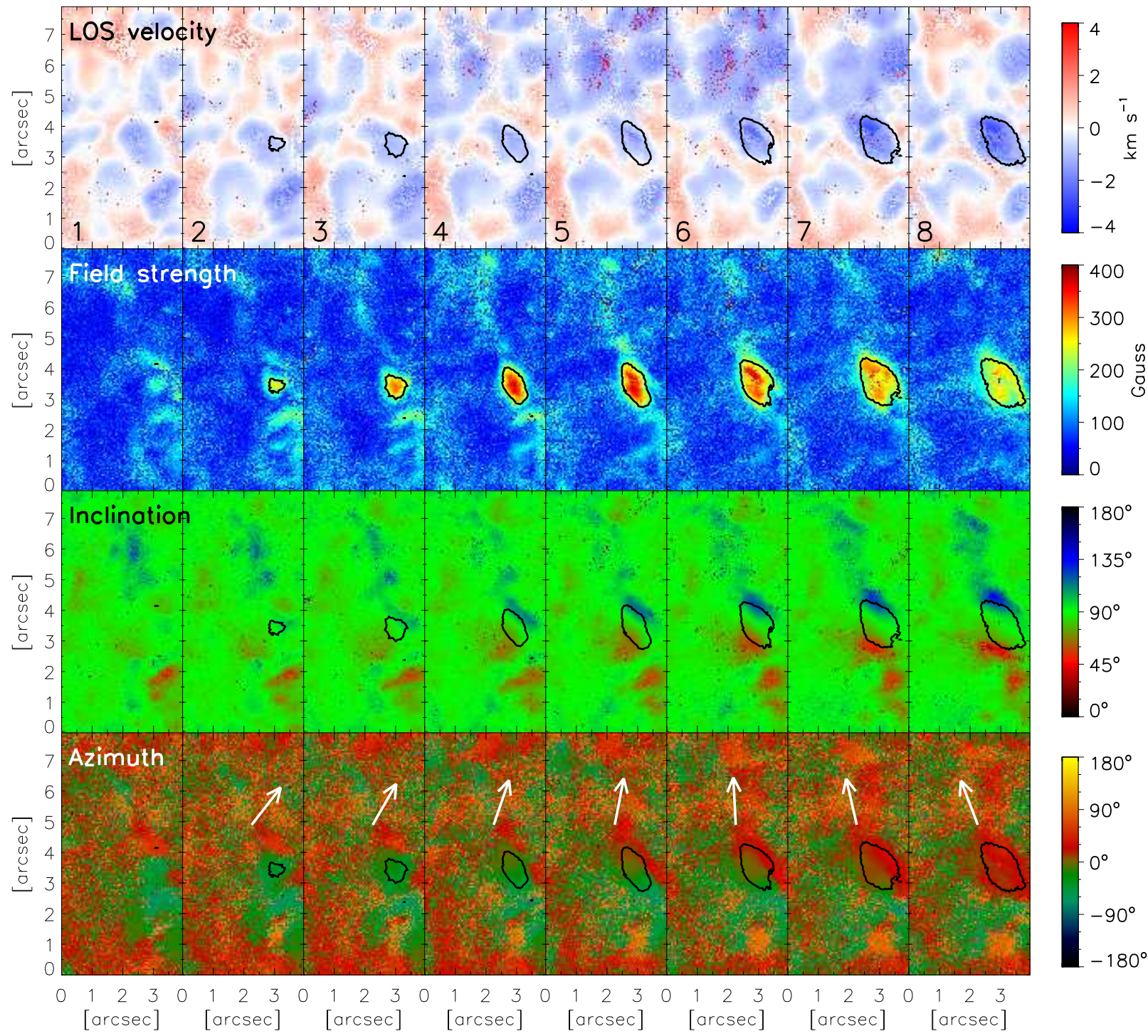

Figure 5. LOS velocity and magnetic field strength at $\log \tau=-2$ (first and second rows), inclination and azimuth angles (third and fourth rows), for frames $1-8$ (compare with Fig. 3). Contours represent a linear polarization signal of $0.5 \%$ of the $I_{c}$, as in Fig. 3 Arrows indicate the mean value of azimuth angle $\phi$ within the $L_{s}$ countour for each frame. The direction to the top of the plot is assumed as zero reference for $\phi$.

the evolution of the bipole after the emergence process: since the evolution during this phase is less rapid, only 


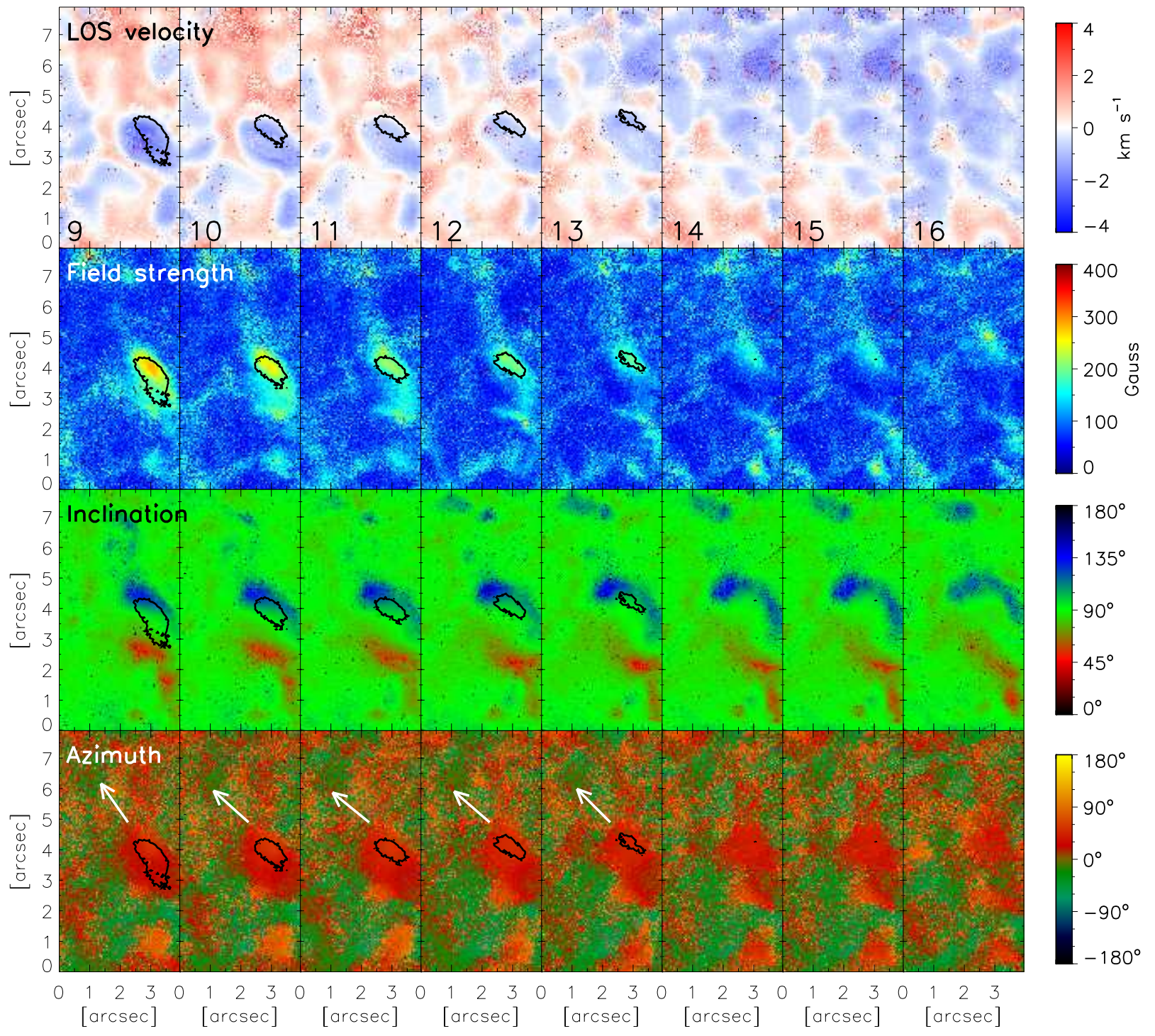

Figure 6. Same as in Fig. 5 for frames $9-16$.

every other frame is shown (but see also the on-line material). Footpoints move apart until they start breaking into smaller flux patches and interact with the surrounding fields. Their remnants remain distinguishable at least for 15 minutes after the first detection of the magnetic structure. In frames $16-18$, the bulk of the negative polarity starts being fragmented, losing its compactness. During this process the various patches of this polarity move along intergranular lanes.

In frames $32-34$ the negative polarity of the bipole totally merges into a pre-existing flux element of the same polarity, and is no longer distinguishable as an individual flux concentration. This explains why the flux curve for the negative polarity footpoint in the right panel of Fig. 4 stops there.

The positive polarity footpoint at the beginning of the decay phase has merged with the pre-existing positive flux of the bipole at the bottom. Note that, in this case, no net flux is lost as the positive polarity footpoint of the pre-existing bipole is aggregated to the positive polarity of the newly emerged one. This could explain why this cancellation episode is not apparent in the red flux curve of Fig. 4 (right panel). After this cancellation, the newly created positive polarity patch is seen to move near the edge of our field of view with little evident interaction with other fluxes (see Fig. 8). Interestingly, during this time the positive flux patch moves largely over granulation dominated regions instead of intergranular ones.

In order to get an indication of the physical processes occurring during the decay phase, we have fitted the flux evolution curves during the decay period using the MPFIT routine (Markwardt 2009), which performs fits to data with user-defined functions. We have used as a guess function an exponential of the type

$$
f(t)=a+b \cdot e^{\beta t} .
$$

We obtained $\beta_{ \pm}=-2.5 \times 10^{-3} \mathrm{~s}^{-1}$ and $-3.0 \times 10^{-3} \mathrm{~s}^{-1}$ for the positive and negative flux, respectively. This corresponds to a "turbulent" diffusion costant $\eta=L^{2} / \tau$ of the order of $8 \times 10^{2} \mathrm{~km}^{2} \mathrm{~s}^{-1}$, where $\tau=\beta^{-1}$ is the $e$ folding time and $L \approx 0.8$ is the size of the footpoint at 

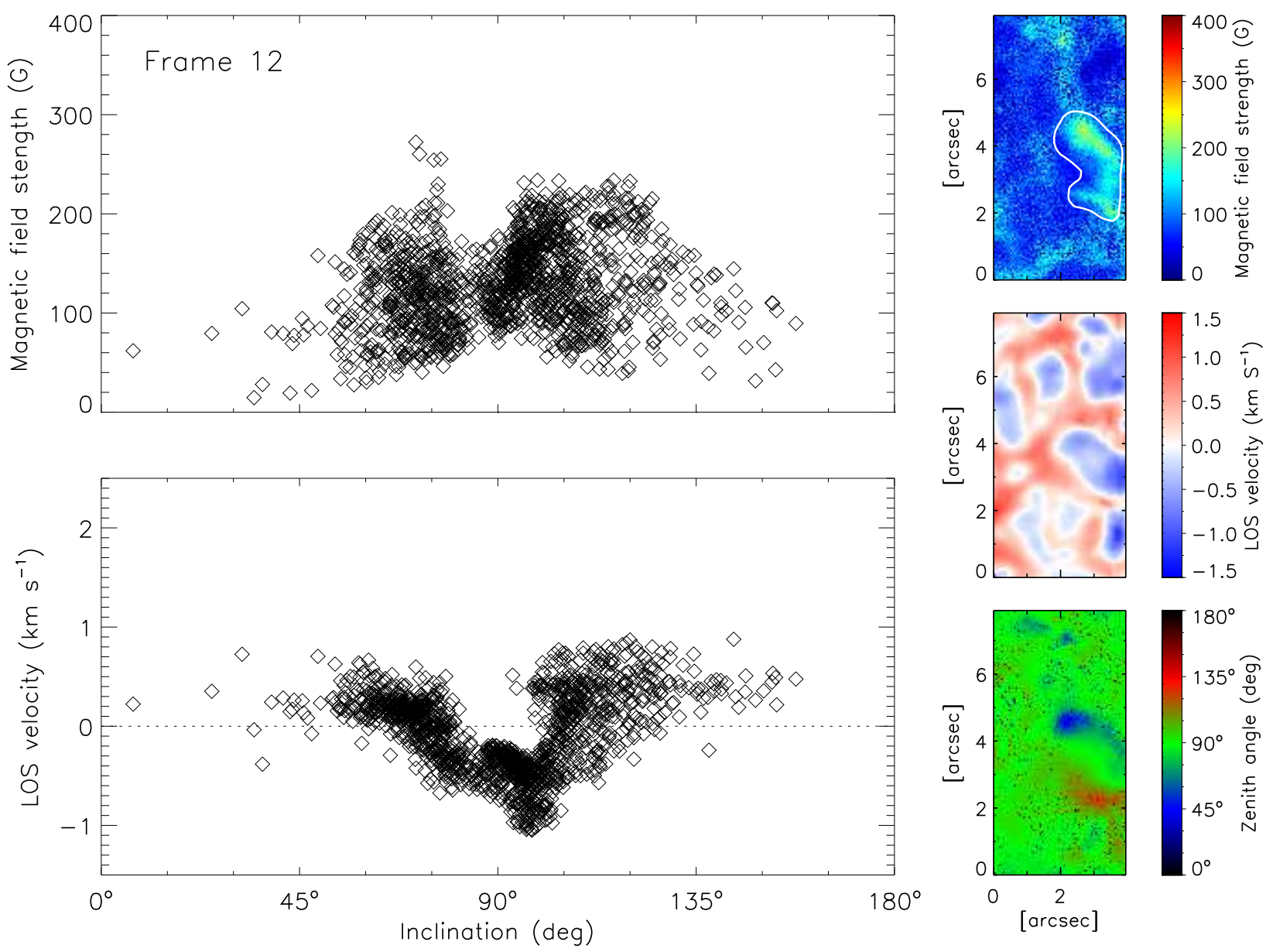

Figure 7. Scatter plots of $B$ and $v_{L O S}$, as a function of the inclination $\gamma$, for a representative frame (No. 12 in Fig. 31 ). We show the results for the SIR inversion without gradients, and the corresponding maps of the physical parameters. Only the pixels within the white contour in the $B$ map with a signal than three times the noise have been indicated in the plot. Keep in mind that inversion have a tendency to retrieve horizontal fields for pixels at noise level.

the time of the flux peak in Fig. 4 (right panel). It is interesting to note that this diffusion constant compares rather well with those inferred by flux transport models of the large scale field, typically set at $6 \times 10^{2} \mathrm{~km}^{2} \mathrm{~s}^{-1}$ (see the instructive review by Sheelev 2005).

This order of magnitude agreement between the diffusion constants of the large scale field and of our granular scale bipole is rather illuminating. As the flux loss occurs in situ, at a rate similar to that encountered in flux transport models, this could indicate an analogous physical process acting in both flux loss scenarios.

Even though the flux decay curve is clearly non-linear, it is interesting to compute a mean flux decay rate which turns out to be $2.86 \times 10^{14} \mathrm{Mx} \mathrm{s}^{-1}$. This rate is one order of magnitude smaller than the flux growth rate. A similar asymmetry between the growth and decay rates is known to exist for active regions (Martínez Pillet 2002; van Driel-Gesztelyi 2002).

\section{DISCUSSION AND CONCLUSIONS}

IMaX/Sunrise observations of the solar photosphere taken at disk center have revealed a number of smallscale episodes of magnetic flux emergence (see, e.g., Danilovic et al. 2010; Solanki et al. 2010, and references therein). We have carried out an analysis of the emer- gence and disappearance of a small magnetic bipole. We have analyzed the polarization maps and then we have inverted the Stokes profiles with the SIR code, to obtain information on the physical parameters of this magnetic structure.

The detection of weak field strengths (400 G) at emergence with horizontal inclination, associated with blueshifted Stokes line profiles between opposite polarity Stokes V profiles, is a well-known signature of the emergence of a concentrated flux loop, as firstly pointed out by Lites et al. (1996) at active region scales. At smaller (granular) scales, our analysis shows results resembling those found by Gömöry et al. (2010), but with a higher cadence and better spatial resolution.

The magnetic flux content places this small bipole at a halfway point between the ephemeral regions studied by Hagenaar (2001) and the all-pervasive loops of the quiet Sun. The bipole indeed appears to be coincident with a granule in the continuum map, so it would apparently represent a typical case of flux emergence at granular scale. However, the present case is the largest structure observed to emerge in the IMaX/Sunrise data analyzed so far.

Once emerged, the magnetic flux spreads over an area 

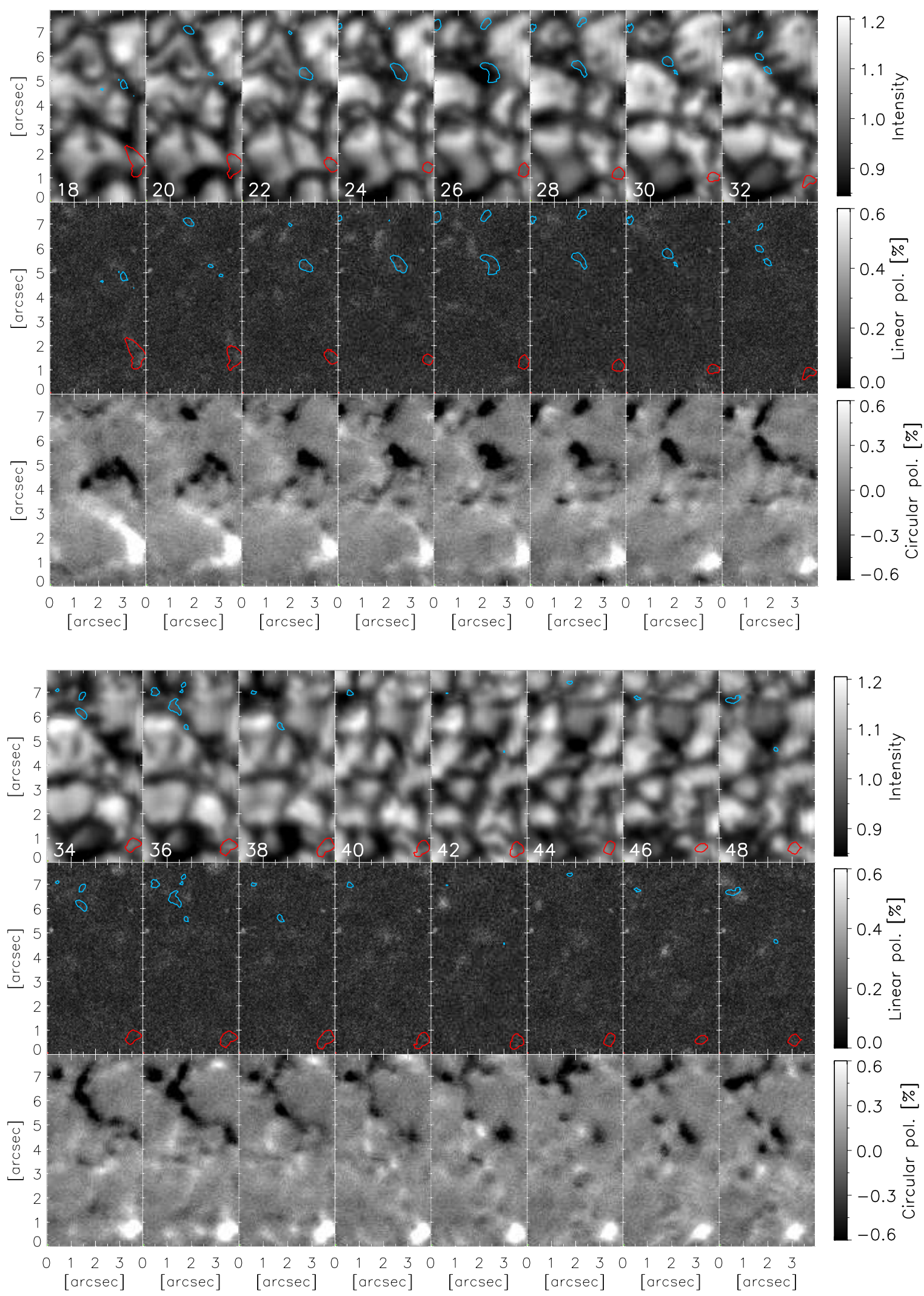

Figure 8. Same as in Fig. 3 but during the decay phase of the bipole. Frame numbering continues from the last frame of Fig. 3 In this figure, the elapsed time between consecutive frames is $66 \mathrm{~s}$. 
larger than the source granule. The footpoints separate up to $4.5 \mathrm{Mm}$ from each other and their remnants remain visible until $15-20$ minutes after the first detection of the magnetic structure. Nevertheless, the granulation pattern seems not to be affected by the flux brought to the surface by the emerging bipole. This agrees with the simulations of Cheung et al. (2007), showing that flux tubes carrying less than $\sim 10^{18} \mathrm{Mx}$ of longitudinal flux do not produce visible disturbances to the granulation pattern.

During the emergence, the footpoints of the bipole do not have the more typical circular shape seen in Hinode observations (e.g., Martínez González \& Bellot Rubio 2009). They rather surround the granule and have a crescent-shaped appearance. Moreover, footpoints do not reach a vertical orientation: they form an angle of about $45^{\circ}$. This could suggest that only a part of the $\Omega$-loop is observed above the continuum formation layer, as also found by Ishikawa et al. (2010), or that the field lines follow the borders of the granule, which could not be vertically steep. Another possibility would be that the field in the footpoint of this emerging loop is not resolved at the spatial resolution ot IMaX level 1 data. Former measurements presented by Solanki et al. (1996) showed that quiet-Sun feature with an amount of flux over $10^{17}$ $\mathrm{Mx}$ tend to have $\mathrm{kG}$ field strength, while the inversions retrieve a field strength of $\approx 250 \mathrm{G}$. An underestimate of the field strength implies that the inclination angle $\gamma$ is overestimated (Stenflo 1985a b) , so that the magnetic field in the footpoints would be more vertical than the inversions indicate. Anyway, such a difference of more than a factor of 3 in the field strength is rather difficult to conceive for our measurements based on the high Zeeman sensitive Fe I $525.02 \mathrm{~nm}$ line $(g=3)$ displaying Stokes $Q$ and $U$ signals above the noise level (see, e.g., frame 12 in Fig. 3). These signals help inferring the field strength in the inversions more reliably than when only Stokes $V$ is used.

The azimuth angle changes during the emergence by about $90^{\circ}$ (see Figs. 5 and 6), in the same direction as the line joining the footpoints. Convective motions acting on the emerging loop or the unfolding of any twist present in the original structure could generate this behaviour.

The flux decay follows an exponential law. The observed cancellation and merging with a pre-existing bipole does not have an effect on the flux history of the bipole and is not the dominant decay process in this example, although it might change the connectivity of the flux patches. Flux removal dominates from the moment in which the maximum flux is observed. Clearly, flux is destroyed in situ, i.e., without visible interactions with opposite polarity patches, in some efficient way that dominates the flux budget as soon as no more flux is brought to the surface. This elusive process probably acts also during the flux emergence phase, but is not evident at this stage simply because emergence is one order of magnitude stronger than the flux decay rate. The diffusion constant estimated for this process is only a little bit higher than typical estimates used for large scale flux transport models $\left(6 \times 10^{2} \mathrm{~km}^{2} \mathrm{~s}^{-1}\right)$. Cameron et al. (2011) have also found similar values in numerical simulations of small-scale mixed polarity field in the nearsurface layers of the Sun. They show that magnetic el- ements are advected by the horizontal granular motions against each other and that the flux is removed at a rate corresponding to an effective turbulent diffusivity of about $3 \times 10^{2} \mathrm{~km}^{2} \mathrm{~s}^{-1}$, which is only slightly smaller than our estimate.

The existence of flux-removing diffusive processes that are similarly effective at active region and granular scales deserves to be further studied from a theoretical and observational point of view. Our value of the diffusion constant can be used to estimate the time in which an active region would decay. As Mever et al. (1974) inferred for the turbulent diffusion of a monolithic magnetic flux concentration, the decay time is given by

$$
t=\frac{\Phi}{4 \pi \eta B_{e}}
$$

where $\Phi$ is the original flux content, $\eta$ the turbulent diffusivity, and $B_{e}$ the equipartition field strength. Using this formula for a typical active region of $10^{22} \mathrm{Mx}$ with our value of the turbulent diffusivity $\left(8 \times 10^{2} \mathrm{~km}^{2} \mathrm{~s}^{-1}\right)$, we estimate a decay time of $\approx 3$ days. As active regions live longer, it seems clear that the diffusivity found in this paper is more effective on smaller flux structures, or possibly on flux structures embedded in the vigorous granular convection found in the quiet Sun, rather than in the smaller and more slowly evolving abnormal granulation in active regions.

Finally, it is interesting to compare the emergence rate with the distribution of emerging fluxes proposed by Thornton \& Parnell (2011). The number of events per unit area per unit time within a given flux range $\Phi$ is

$$
N_{\mathrm{ev}}(\Phi)=\left.\frac{n_{0}}{2-\alpha}\left(\frac{\Phi}{\Phi_{0}}\right)^{1-\alpha}\right|_{\Phi 1} ^{\Phi_{2}},
$$

where $n_{0}=3.14 \times 10^{-14} \mathrm{~cm}^{-2}$ day $^{-1}$ and $\Phi_{0}=10^{16} \mathrm{Mx}$. Remembering that they found a power index $\alpha=-2.7$, we obtain a frequency of $N_{\mathrm{ev}}=4.9 \times 10^{-17} \mathrm{~cm}^{-2}$ day $^{-1}$ for a flux range from $5.5 \times 10^{17}$ to $1 \times 10^{20} \mathrm{Mx}$. Taking into account our $\mathrm{FoV}$ of $\simeq 1300 \mathrm{Mm}^{2}$ and the duration of the observations of 30 minutes, we get $N_{\mathrm{ev}} \simeq 14$ events (i.e., 7 bipoles). The observed number of flux patches within this range in our data set is smaller than the prediction from the Thornton \& Parnell (2011) work, as we found only the two footpoints of the bipole. Note also that no flux patches with fluxes above $6.5 \times 10^{17} \mathrm{Mx}$ are observed, whereas the above distribution would have suggested otherwise. Given the short duration of the period analysed here, it is unclear whether this disagreement is fortuitous or indicates an excess in the predicted number of bipoles due to an overestimate of the total density of emergence events. The activity minimum in which the Sun was residing during the SUNRISE flight could also be behind our low number of observed bipoles. Note that the difference in both time and space resolution between the two data sets could affect this comparison: thus, it would be worthwhile applying the algorithm used by Thornton \& Parnell (2011) to these SunRISE observations.

On the other hand, the bipole closely behaves as a small-scale version of an ER: we found a good agreement with the dependence of the footpoint separation with the 
flux content proposed by Hagenaar (2001). The overall morphology is quite similar, even if we do not find any evidence of mixed polarities in between the two footpoints during the emergence, as reported by Guglielmino et al. (2010) for an ER with flux content $\sim 1.5 \times 10^{19} \mathrm{Mx}$, i.e., twenty times larger than the bipole studied here.

Further studies of similar time series can be of great value to consolidate the results presented here. More cases are needed to clarify these findings, using spectropolarimetric data of a similar resolution, sensitivity and temporal coverage such as those that could be obtained with specially tailored observations with SOT/Hinode, CRISP/SST, and IBIS/DST. We are not aware of such results yet and, thus, it is difficult for the moment to extend and compare these findings with more examples, and to understand their implications for the global solar flux budget.

This work has been partially funded by the Spanish Ministerio de Educación y Ciencia, through Projects ESP2006-13030-C06-01/02/03/04 and AYA2009-14105C06, and Junta de Andalucía, through Project P07-TEP2687, including a percentage from European FEDER funds. The German contribution to SunRISE is funded by the Bundesministerium für Wirtschaft und Technologie through Deutsches Zentrum für Luft- und Raumfahrt e.V. (DLR), Grant No. 50 OU 0401, and by the Innovationsfond of the President of the Max Planck Society (MPG). Financial support by the European Commission through the SOLAIRE Network (MTRN-CT2006-035484) is gratefully acknowledged. S.L.G. and L.R.B.R. acknowledge insightful discussions on smallscale flux emergence processes within the ISSI International Team lead by K. Galsgaard and F. Zuccarello at the ISSI (International Space Science Institute, Bern), and the ISSI for support. This work has been partially supported by the WCU grant (No. R31-10016) funded by the Korean Ministry of Education, Science and Technology. Use of NASA's Astrophysical Data System is gratefully acknowledged.

Facilities: Sunrise.

\section{REFERENCES}

Barthol, P., et al. 2011, Sol. Phys., 268, 1

Berkefeld, T., et al. 2011, Sol. Phys., 268, 103

Cameron, R., Vögler, A., \& Schüssler, M. 2011, A\&A, 533, A86

Cattaneo, F. 1999, ApJ, 515, L39

Centeno, R., et al. 2007, ApJ, 666, L137

Cheung, M. C. M., Schüssler, M., \& Moreno-Insertis, F. 2007, A\&A, 467, 703

Danilovic, S., et al. 2011, A\&A, submitted

Danilovic, S., et al. 2010, ApJ, 723, L149

Gandorfer, A., et al. 2011, Sol. Phys., 268, 35

Gingerich, O., Noyes, R. W., Kalkofen, W., \& Cuny, Y. 1971, Sol. Phys., 18, 347
Gömöry, P., Beck, C., Balthasar, H., Rybák, J., Kučera, A., Koza, J., \& Wöhl, H. 2010, A\&A, 511, A14

Guglielmino, S. L., Bellot Rubio, L. R., Zuccarello, F., Aulanier, G., Vargas Domínguez, S., \& Kamio, S. 2010, ApJ, 724, 1083

Hagenaar, H. J., De Rosa, M. L., \& Schrijver, C. J. 2008, ApJ, 678,541

Hagenaar, H. J., Schrijver, C. J., \& Title, A. M. 2003, ApJ, 584, 1107

Hagenaar, H. J. 2001, ApJ, 555, 448

Harvey, K. L., \& Martin, S. F. 1973, Sol. Phys., 32, 389

Harvey, K. L., Harvey, J. W., \& Martin, S. F. 1975, Sol. Phys., 40, 87

Ishikawa, R., Tsuneta, S., \& Jurčák, J. 2010, ApJ, 713, 1310

Ishikawa, R., et al. 2008, A\&A, 481, L25

Kosugi, T., et al. 2007, Sol. Phys., 243, 3

Lites, B. W., Skumanich, A., Martínez Pillet, V. 1998, ApJ, 333, 1053

Lites, B. W., et al. 2008, ApJ, 673, 1237

Lites, B. W., Leka, K. D., Skumanich, A., Martinez Pillet, V., \& Shimizu, T. 1996, ApJ, 460, 1019

Markwardt, C. B. 2009, Astronomical Data Analysis Software and Systems XVIII, Edited by David A. Bohlender, Daniel Durand, and Patrick Dowler. Astronomical Society of the Pacific Conference Series, 411, 251

Martin, S. F. 1988, Sol. Phys., 117, 243

Martin, S. F., \& Harvey, K. H. 1979, Sol. Phys., 64, 93

Martínez González, M. J., \& Bellot Rubio, L. R. 2009, ApJ, 700, 1391

Martínez González, M. J., Collados, M., Ruiz Cobo, B., \& Solanki, S. K. 2007, A\&A, 469, L39

Martínez Pillet, V., et al. 2011, Sol. Phys., 268, 57

Martínez Pillet, V. 2002, Astronomische Nachrichten, 323, 342

Meyer, F., Schmidt, H. U., Wilson, P. R., \& Weiss, N. O. 1974, MNRAS, 169, 35

Moreno Insertis, F., Caligari, P. \& Schüssler, M. 1995, ApJ, 452, 894

Nordlund, A., Brandenburg, A., Jennings, R. L., Rieutord, M., Ruokolainen, J., Stein, R. F., \& Tuominen, I. 1992, ApJ, 392, 647

Orozco Suárez, D., Bellot Rubio, L. R., del Toro Iniesta, J. C., \&

Tsuneta, S. 2008, A\&A, 481, L33

Parker, E. N. 1993, ApJ, 408, 707

Parker, E. N. 1955, ApJ, 122, 293

Parnell, C. E., DeForest, C. E., Hagenaar, H. J., Johnston, B. A., Lamb, D. A., \& Welsch, B. T. 2009, ApJ, 698, 75

Roth, M., et al. 2010, ApJ, 723, L175

Ruiz Cobo, B., \& del Toro Iniesta, J. C. 1992, ApJ, 398, 375

Sheeley, N. R. 2005, Living Rev. Solar Phys., 2, 5

Socas-Navarro, H., \& Sánchez Almeida, J. 2002, ApJ, 565, 1323

Solanki, S. K., et al. 2010, ApJ, 723, L127

Solanki, S. K., Lagg, A., Woch, J., Krupp, N., \& Collados, M. 2003, Nature, 425, 692

Solanki, S. K., Zufferey, D., Lin, H., Rüedi, I., \& Kuhn, J. R. 1996, A\&A, 310, L33

Stein, R. F., Lagerfjärd, A., Nordlund, Å., \& Georgobiani, D. 2010, Sol. Phys., 34

Stenflo, J. O. 1985, Measurements of Solar Vector Magnetic Fields, 263

Stenflo, J. O. 1985, Sol. Phys., 100, 189

Thornton, L. M., \& Parnell, C. E. 2011, Sol. Phys., 269, 13

Tsuneta, S., et al. 2008, Sol. Phys., 249, 167

van Driel-Gesztelyi, L. 2002, SOLMAG 2002. Proceedings of the Magnetic Coupling of the Solar Atmosphere Euroconference, 505,113 